\title{
Physical insight into Timoshenko beam theory and its modification with extension
}

\author{
Ivo Senjanović and Nikola Vladimir* \\ Faculty of Mechanical Engineering and Naval Architecture, University of Zagreb, \\ Ivana Lučića 5, 10000 Zagreb, Croatia
}

(Received July 4, 2013, Revised October 29, 2013, Accepted November 1, 2013)

\begin{abstract}
An outline of the Timoshenko beam theory is presented. Two differential equations of motion in terms of deflection and rotation are comprised into single equation with deflection and analytical solutions of natural vibrations for different boundary conditions are given. Double frequency phenomenon for simply supported beam is investigated. The Timoshenko beam theory is modified by decomposition of total deflection into pure bending deflection and shear deflection, and total rotation into bending rotation and axial shear angle. The governing equations are condensed into two independent equations of motion, one for flexural and another for axial shear vibrations. Flexural vibrations of a simply supported, clamped and free beam are analysed by both theories and the same natural frequencies are obtained. That fact is proved in an analytical way. Axial shear vibrations are analogous to stretching vibrations on an axial elastic support, resulting in an additional response spectrum, as a novelty. Relationship between parameters in beam response functions of all type of vibrations is analysed.
\end{abstract}

Keywords: Timoshenko beam theory; flexural vibration; axial shear vibration; vibration parameter; analytical solution; double frequency phenomenon

\section{Introduction}

Beam is used as a structural element in many engineering structures like frame and grillage ones (Pilkey 2002, Pavazza 2007, Carrera et al. 2011). Moreover, the whole complex structure can be modelled as a beam to some extend like ship hulls, floating airports, etc (Senjanović et al. 2009). The Euler-Bernoulli theory is widely used for simulation of a slender beam behaviour. For thick beam Timoshenko theory has been developed by taking shear influence and rotary inertia into account (Timoshenko 1921, 1922). Shear effect is extremely large in higher vibration modes due to reduced mode half wave length.

The Timoshenko beam theory deals with two differential equations of motion with deflection and cross-section rotation as the basic variables (Timoshenko 1921, 1922). The system is reduced into a single four order partial differential equation by Timoshenko (1937), where only approximate solutions are given as commented in (Inman 1994) and (van Rensburg and van der Merve 2006). In the most papers the first approach with two differential equations is used in order to ensure control of exact and complete beam behaviour, (Geist and McLaughlin 1997, van

*Corresponding author, Ph.D., E-mail: nikola.vladimir@fsb.hr 
Rensburg and van der Merve 2006). Possibility to operate with single equation of motion in terms of pure bending deflection is noticed and recently used, due to reason of simplicity, as an approximated but reliable enough solution (Senjanović et al. 1989, Li 2008).

The Timoshenko beam theory is applied as a base for more complex problems, like beam vibrations on elastic foundation (De Rosa 1995), beam vibrations and buckling on elastic foundation (Matsunaga 1999), vibrations of double-beam system with transverse and axial load (Stojanović and Kozić 2012), vibration and stability of multiple beam systems (Stojanović et al. 2013), beam response moving to load (Sniady 2008), etc. Recently, the Timoshenko beam theory is used in nanotechnology for vibration analysis of nanotubes, as for instance (Simsek 2011). Timoshenko idea of shear and rotary inertia influence on deflection is not only limited to beams. These effects are also incorporated in the Mindlin thick plate theory as a 2D problem (Mindlin 1951). Timoshenko beam static functions are often used as coordinate functions for thick plate vibration analysis by the Rayleigh-Ritz method (Zhou 2001). Furthermore, differential equation of beam torsion, with shear influence is based on analogy with that for beam bending (Pavazza 2005). Hence, in case of coupled flexural and torsional vibrations of a girder with open crosssection the same mathematical model is used for analysis of both responses (Senjanović et al. 2009).

The Timoshenko beam theory plays an important role in development of sophisticated beam finite elements. Various finite elements have been worked out in the last decades. They are distinguished in the choice of interpolation functions for mathematical description of deflection and rotation. Application of the same order polynomials leads to so-called shear locking, since bending strain energy for a slender beam vanishes before shear strain energy. If static solution of Timoshenko beam is used for deflection and rotation functions this problem is overcome (Reddy 1997, Senjanović et al. 2009).

In spite of the fact that many papers have been published on Timoshenko beam theory during long period of time, it seams that all phenomena hidden in that theory are not yet investigated.

Motivated by the state-of-the art, some additional investigation has been undertaken and the obtained results presented in this paper shed more light on the considered subject. In Section 2 an outline of the Timoshenko beam theory is presented, where basic equations in terms of deflection and cross-section rotation are listed, and general solution for natural vibrations is given. In Section 3 the Timoshenko beam theory is modified in such a way that deflection is split into pure bending deflection and shear deflection, while rotation is decomposed into cross-section rotation due to pure bending and axial shear angle, as a novelty. Application of both theories is illustrated in Section 4 within numerical examples for simply supported, clamped and free beam. In Section 5 comparison of the theories is done. It is found that flexural part of the modified beam theory, used in the literature as an approximate alternative, is actually rigorous as that based on the original theory. Axial shear vibrations extracted from the Timoshenko beam theory, gives an additional natural frequency spectrum.

In Appendix A frequency equations for clamped and free Timoshenko beam are specified, and in Appendix B the same is done for the modified beam theory. Linear relation between the above frequency equations is presented in Section 5. A detail analysis of vibration parameters in arguments of hyperbolic and trigonometric functions in solutions of beam response is performed in Appendix C. Their exact asymptotic values as function of frequency are specified, that is an improvements comparing to the known approximate values. It is confirmed that double frequency spectrum is phenomenon related only to the simply supported beam. In that way dilemma concerning this subject is overcome. In Section 6 valuable conclusions based on the performed detail analysis are drawn. 


\section{Timoshenko beam theory}

\subsection{Basic equations}

Timoshenko beam theory deals with beam deflection and angle of rotation of cross-section, $w$ and $\psi$, respectively (Timoshenko 1921, 1922). The sectional forces, i.e., bending moment and shear force read

$$
M=D \frac{\partial \psi}{\partial x}, \quad Q=S\left(\frac{\partial w}{\partial x}+\psi\right)
$$

where $D=E I$ is flexural rigidity and $S=k G A$ is shear rigidity, $A$ is cross-section area and $I$ is its moment of inertia, $k$ is shear coefficient, and $E$ and $G=E /(2(1+v))$ is Young's modulus and shear modulus, respectively. Value of shear coefficient depends on beam cross-section profile (Cowper 1966, Senjanović and Fan 1990). Stiffness properties for complex thin-walled girder are determined by the strip element method (Senjanović and Fan 1993).

Beam is loaded with transverse inertia load per unit length, and distributed bending moment

$$
q_{x}=-m \frac{\partial^{2} w}{\partial t^{2}}, \quad m_{x}=-J \frac{\partial^{2} \psi}{\partial t^{2}}
$$

where $m=\rho A$ is specific mass per unit length and $J=\rho I$ is its moment of inertia.

Equilibrium of moments and forces

$$
\frac{\partial M}{\partial x}-Q=-m_{x}, \quad \frac{\partial Q}{\partial x}=-q_{x}
$$

leads to two coupled differential equations

$$
\begin{gathered}
D \frac{\partial^{2} \psi}{\partial x^{2}}-S\left(\frac{\partial w}{\partial x}+\psi\right)-J \frac{\partial^{2} \psi}{\partial t^{2}}=0 \\
S\left(\frac{\partial^{2} w}{\partial x^{2}}+\frac{\partial \psi}{\partial x}\right)-m \frac{\partial^{2} w}{\partial t^{2}}=0
\end{gathered}
$$

From (5) yields

$$
\frac{\partial \psi}{\partial x}=-\frac{\partial^{2} w}{\partial x^{2}}+\frac{m}{S} \frac{\partial^{2} w}{\partial t^{2}}
$$

and by substituting (6) into (4) derived per $x$, one arrives at the single beam differential equation of motion

$$
\frac{\partial^{4} w}{\partial x^{4}}-\left(\frac{J}{D}+\frac{m}{S}\right) \frac{\partial^{4} w}{\partial x^{2} \partial t^{2}}+\frac{m}{D} \frac{\partial^{2}}{\partial t^{2}}\left(w+\frac{J}{S} \frac{\partial^{2} w}{\partial t^{2}}\right)=0
$$

Once (7) is solved angle of rotation is obtained from (6) as

$$
\psi=-\frac{\partial w}{\partial x}+\frac{m}{S} \int \frac{\partial^{2} w}{\partial t^{2}} \mathrm{~d} x+f(t)
$$


where $f(t)$ is rigid body motion.

If $w$ is extracted from (4) and substituted in (5) the same type of differential equation as (7) is obtained for $\psi$ and (8) for $w$.

\subsection{General solution of natural vibrations}

In natural vibrations $w=W \sin \omega t$ and $\psi=\Psi \sin \omega t$, and Eqs. (7) and (8) are reduced to the vibration amplitudes

$$
\begin{gathered}
\frac{\mathrm{d}^{4} W}{\mathrm{~d} x^{4}}+\omega^{2}\left(\frac{J}{D}+\frac{m}{S}\right) \frac{\mathrm{d}^{2} W}{\mathrm{~d} x^{2}}+\omega^{2} \frac{m}{D}\left(\omega^{2} \frac{J}{S}-1\right) W=0 \\
\Psi=-\frac{\mathrm{d} W}{\mathrm{~d} x}-\omega^{2} \frac{m}{S} \int W \mathrm{~d} x+C .
\end{gathered}
$$

Solution of (9) can be assumed in the form $W=A e^{\gamma x}$ that leads to biquadratic equation

$$
\gamma^{4}+a \gamma^{2}+b=0
$$

where

$$
a=\omega^{2}\left(\frac{J}{D}+\frac{m}{S}\right), \quad b=\omega^{2} \frac{m}{D}\left(\omega^{2} \frac{J}{S}-1\right) .
$$

Roots of (11) read

$$
\gamma=\alpha,-\alpha, i \beta,-i \beta
$$

where $i=\sqrt{-1}$ and

$$
\begin{aligned}
& \alpha=\frac{\omega}{\sqrt{2}} \sqrt{\sqrt{\left(\frac{m}{S}-\frac{J}{D}\right)^{2}+\frac{4 m}{D \omega^{2}}}-\left(\frac{m}{S}+\frac{J}{D}\right)} \\
& \beta=\frac{\omega}{\sqrt{2}} \sqrt{\sqrt{\left(\frac{m}{S}-\frac{J}{D}\right)^{2}+\frac{4 m}{D \omega^{2}}}+\left(\frac{m}{S}+\frac{J}{D}\right)} .
\end{aligned}
$$

Deflection function with its derivatives and the first integral can be presented in the matrix form

$$
\left\{\begin{array}{c}
W \\
W^{\prime} \\
W^{\prime \prime} \\
W^{\prime \prime \prime} \\
\int W \mathrm{~d} x
\end{array}\right\}=\left[\begin{array}{cccc}
\operatorname{sh} \alpha x & \operatorname{ch} \alpha x & \sin \beta x & \cos \beta x \\
\alpha \operatorname{ch} \alpha x & \alpha \operatorname{sh} \alpha x & \beta \cos \beta x & -\beta \sin \beta x \\
\alpha^{2} \operatorname{sh} \alpha x & \alpha^{2} \operatorname{ch} \alpha x & -\beta^{2} \sin \beta x & -\beta^{2} \cos \beta x \\
\alpha^{3} \operatorname{ch} \alpha x & \alpha^{3} \operatorname{sh} \alpha x & -\beta^{3} \cos \beta x & \beta^{3} \sin \beta x \\
\frac{1}{\alpha} \operatorname{ch} \alpha x & \frac{1}{\alpha} \operatorname{sh} \alpha x & -\frac{1}{\beta} \cos \beta x & \frac{1}{\beta} \sin \beta x
\end{array}\right]\left\{\begin{array}{c}
A_{1} \\
A_{2} \\
A_{3} \\
A_{4}
\end{array}\right\} .
$$

According to the solution of Eq. (9), Eq. (10) and Eq. (1), beam displacements and forces read 


$$
\begin{gathered}
W=A_{1} \operatorname{sh} \alpha x+A_{2} \operatorname{ch} \alpha x+A_{3} \sin \beta x+A_{4} \cos \beta x \\
\Psi=-\left[\left(1+\frac{\omega^{2} m}{\alpha^{2} S}\right) \alpha A_{1} \operatorname{ch} \alpha x+\left(1+\frac{\omega^{2} m}{\alpha^{2} S}\right) \alpha A_{2} \operatorname{sh} \alpha x+\left(1-\frac{\omega^{2} m}{\beta^{2} S}\right) \beta A_{3} \cos \beta x-\left(1-\frac{\omega^{2} m}{\beta^{2} S}\right) \beta A_{4} \sin \beta x\right] \\
M=-D\left[\left(\alpha^{2}+\omega^{2} \frac{m}{S}\right)\left(A_{1} \operatorname{sh} \alpha x+A_{2} \operatorname{ch} \alpha x\right)-\left(\beta^{2}-\omega^{2} \frac{m}{S}\right)\left(A_{3} \sin \beta x+A_{4} \cos \beta x\right)\right] \\
Q=-\frac{\omega^{2} m}{\alpha \beta}\left(\beta A_{1} \operatorname{ch} \alpha x+\beta A_{2} \operatorname{sh} \alpha x-\alpha A_{3} \cos \beta x+\alpha A_{4} \sin \beta x\right) .
\end{gathered}
$$

Relative values of constants $A_{i}, i=1,2,3,4$, are determined by satisfying four boundary conditions. Since there is no additional condition constant $C$ in (10) is ignored.

$$
\text { Coefficient } \alpha \text {, Eq. (14), can be zero, in which case } \omega_{0}=\sqrt{S / J} \text { and }
$$

$\beta_{0}=\sqrt{(S / D)+(m / J)}$. Deflection function according to (17) takes the form

$$
W=A_{1} x+A_{2}+A_{3} \sin \beta_{0} x+A_{4} \cos \beta_{0} x
$$

where the first two terms describe rigid body motion. If $\omega>\omega_{0}$, then $\alpha=i \tilde{\alpha}$, where

$$
\tilde{\alpha}=\frac{\omega}{\sqrt{2}} \sqrt{\left(\frac{m}{S}+\frac{J}{D}\right)-\sqrt{\left(\frac{m}{S}-\frac{J}{D}\right)^{2}+\frac{4 m}{D \omega^{2}}}}
$$

and deflection function reads

$$
W=A_{1} \sin \tilde{\alpha} x+A_{2} \cos \tilde{\alpha} x+A_{3} \sin \beta x+A_{4} \cos \beta x .
$$

Expressions for displacements and forces Eqs. (17)-(20) have to be transformed accordingly. Hence, $\operatorname{ch} \alpha x=\cos \tilde{\alpha} x, \operatorname{sh} \alpha x=i \sin \tilde{\alpha} x$, where imaginary unit is included in constant $A_{1}$, $\alpha^{2}=-\tilde{\alpha}^{2}$, instead of single factor $\alpha$ it is necessary to write $\tilde{\alpha}$, and finally all functions associated with $A_{1}$ and $A_{2}$ must have the same sign as those with $A_{3}$ and $A_{4}$.

The above analysis shows that beam has a lower and higher spectral response, and transition one. Frequency spectra are shifted for threshold frequency $\omega_{0}$. This problem is also investigated in (Geist and McLaughlin 1997, van Rensburg and van der Merve 2006, Li 2008). The basic differential Eqs. (4) and (5) are solved in (van Rensburg and van der Merve 2006) by assuming solution in the form $w=A e^{\gamma x}$ and $\psi=B e^{\gamma x}$ and the same expressions for displacements (17) and (18) are obtained.

\subsection{Simply supported beam}

Origin of the coordinate system is located in the middle of beam length due to reason of simplicity. Symmetric natural modes for lower frequency spectrum are considered for which constant $A_{1}=A_{3}=0$. Boundary conditions read $W(l / 2)=0$ and $M(l / 2)=0$, and one obtains from (18) and (19) system of equations 


$$
\left[\begin{array}{cc}
\operatorname{ch} \alpha \frac{l}{2} & \cos \beta \frac{l}{2} \\
-\left(\alpha^{2}+\omega^{2} \frac{m}{S}\right) \operatorname{ch} \alpha \frac{l}{2} & \left(\beta^{2}-\omega^{2} \frac{m}{S}\right) \cos \beta \frac{l}{2}
\end{array}\right]\left\{\begin{array}{l}
A_{2} \\
A_{4}
\end{array}\right\}=\left\{\begin{array}{l}
0 \\
0
\end{array}\right\} .
$$

Its determinant has to be equal to zero for non-trivial solution

$$
\operatorname{Det}=\left(\alpha^{2}+\beta^{2}\right) \operatorname{ch} \alpha \frac{l}{2} \cos \beta \frac{l}{2}=0 .
$$

The above frequency equation is satisfied if $\beta l / 2=(2 n-1) \pi / 2$. By employing expression (15) for $\beta$, yields

$$
\omega_{n}^{4}-a_{n} \omega_{n}^{2}+b_{n}=0
$$

where

$$
\begin{gathered}
a_{n}=\frac{S}{J}+\left(\frac{D}{J}+\frac{S}{m}\right) \beta_{n}^{2} \\
b_{n}=\frac{D S}{J m} \beta_{n}^{4}, \quad \beta_{n}=\frac{(2 n-1) \pi}{l}, \quad n=1,2 \ldots
\end{gathered}
$$

Two positive solutions of (26) read

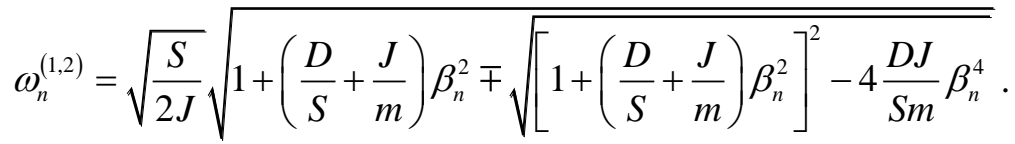

They characterize the first and the second frequency spectrum, respectively. Relative values of integration constants can be determined from the first of Eq. (24)

$$
A_{2}^{(n)}=\cos \beta_{n} \frac{l}{2}=0, \quad A_{4}^{(n)}=-\operatorname{ch} \alpha_{n} \frac{l}{2} .
$$

Since one constant is zero, another is arbitrary and natural modes read $W_{n}=A_{n} \cos ((2 n-1) \pi x / l)$, $n=1,2, \ldots$

If $\omega>\omega_{0}$ than frequency equation (25) is transformed into

$$
\left(\beta^{2}-\tilde{\alpha}^{2}\right) \cos \tilde{\alpha} \frac{l}{2} \cos \beta \frac{l}{2}=0 .
$$

Now $\tilde{\alpha}_{n} l / 2=(2 n-1) \pi / 2$ and by employing (22) for $\tilde{\alpha}$ the same expression for natural frequencies as in the previous case is obtained, i.e., Eq. (29). Ratio of the integration constants is

$$
\frac{A_{4}^{(n)}}{A_{2}^{(n)}}=-\frac{\cos \beta_{n} \frac{l}{2}}{\cos \tilde{\alpha}_{n} \frac{l}{2}}=\frac{0}{0}
$$


and both constants are arbitrary, that results in the common natural modes $W_{n}=A_{n} \cos ((2 n-1) \pi x / l)$, where $n>n_{0}, n_{0}=\beta_{0} l / \pi$.

Hence, for an integer $n$ two frequency spectra exist, one due to $\beta_{n}$ and another due to $\tilde{\alpha}_{n}$, which are shifted for $\omega_{0}$. Since $\beta_{n} l / 2=\tilde{\alpha}_{n} l / 2$ their natural modes are identical.

In similar way eigenpairs for antisymmetric modes taking $A_{2}=A_{4}=0$ into account, can be determined. In that case $\sin \beta_{n} l / 2=0$ and $\sin \tilde{\alpha}_{n} l / 2=0$, that requires $\beta_{n} l / 2=\tilde{\alpha}_{n} l / 2=n \pi, n=1,2 \ldots$ Formula (29) for natural frequencies is valid with $\beta_{n}=\tilde{\alpha}_{n}=2 n \pi / l$. Integration constants are expressed with $s h$ and $\sin$ functions in an previously analogous way.

Natural frequencies can be also directly determined from differential Eq. (9) by assuming natural modes in the form $W_{n}=A_{n} \sin (n \pi x / l)$. Formula (29) is obtained with $\beta_{n}=n \pi / l, n=1,2 \ldots$ that includes both symmetric $(n=1,3 \ldots)$ and antisymmetric $(n=2,4 \ldots)$ modes.

Double frequency phenomenon is analysed in (van Rensburg and van der Merve 2006), starting from basic Eqs. (4) and (5) with two variables, and the same results as presented above are obtained.

\subsection{Clamped beam}

Symmetric natural modes are considered, taking $A_{1}=A_{3}=0$. Boundary conditions read $W(l / 2)=0$ and $\Psi(l / 2)=0$ and one obtains by employing Eqs. (18) and (19) frequency equation for lower spectrum (A1) shown in Appendix A. The integration constants are represented with Eq. (30). Frequency equation for antisymmetric modes is obtained by taking constants $A_{2}=A_{4}=0$, Eq. (A2). In similar way frequency equations for symmetric and antisymmetric modes for higher spectrum are specified, Eqs. (A3) and (A4), respectively.

\subsection{Free beam}

In this case boundary conditions read $M(l / 2)=0$ and $Q(l / 2)=0$. Frequency equations for lower and higher spectrum, and symmetric and antisymmetric modes, are also given in Appendix A, Eqs. (A5), (A6), (A7) and (A8), respectively.

\section{Modified beam theory}

\subsection{Differential equations of motion}

Beam deflection $w$ and angle of rotation $\psi$ are split into their constitutive parts, Fig. 1, i.e.

$$
w=w_{b}+w_{s}, \quad \psi=\varphi+\vartheta, \quad \varphi=-\frac{\partial w_{b}}{\partial x},
$$

where $w_{b}$ and $w_{s}$ is beam deflection due to pure bending and transverse shear, respectively, and $\varphi$ is angle of cross-section rotation due to bending, while $\vartheta$ is cross-section slope due to axial shear. Equilibrium Eqs. (4) and (5) can be presented in the form with the separated variables $w_{b}$ and $w_{s}$, and $\vartheta$ 
a)

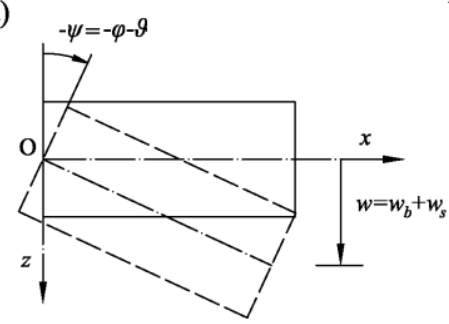

c)

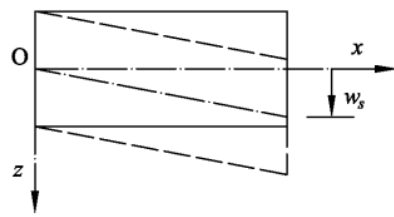

b)

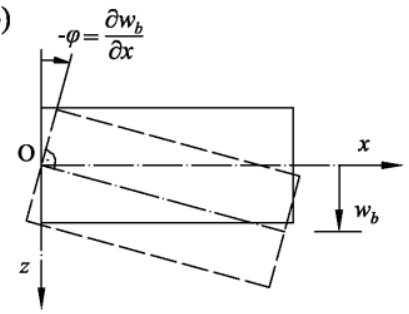

d)

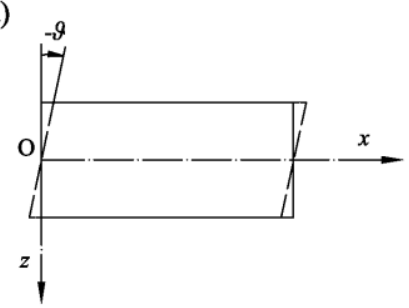

Fig. 1 Thick beam displacements (a) total deflection and rotation $w, \psi$, (b) pure bending deflection and rotation $w_{b}, \varphi,(\mathrm{c})$ transverse shear deflection $w_{s},(\mathrm{~d})-$ axial shear angle $\vartheta$

$$
\begin{gathered}
D \frac{\partial^{3} w_{b}}{\partial x^{3}}-J \frac{\partial^{2}}{\partial t^{2}}\left(\frac{\partial w_{b}}{\partial x}\right)+S \frac{\partial w_{s}}{\partial x}=D \frac{\partial^{2} \vartheta}{\partial x^{2}}-S \vartheta-J \frac{\partial^{2} \vartheta}{\partial t^{2}} \\
S \frac{\partial^{2} w_{s}}{\partial x^{2}}-m \frac{\partial^{2}}{\partial t^{2}}\left(w_{b}+w_{s}\right)=-S \frac{\partial \vartheta}{\partial x} .
\end{gathered}
$$

Since only two equations are available for three variables one can assume that flexural and axial shear displacement fields are not coupled. In that case, by setting both left and right hand side of (34) zero, yields from the former

$$
w_{s}=-\frac{D}{S} \frac{\partial^{2} w_{b}}{\partial x^{2}}+\frac{J}{S} \frac{\partial^{2} w_{b}}{\partial t^{2}} .
$$

By substituting (36) into (35) differential equation for flexural vibrations is obtained, which is expressed with pure bending deflection

$$
\frac{\partial^{4} w_{b}}{\partial x^{4}}-\left(\frac{J}{D}+\frac{m}{S}\right) \frac{\partial^{4} w_{b}}{\partial x^{2} \partial t^{2}}+\frac{m}{D} \frac{\partial^{2}}{\partial t^{2}}\left(w_{b}+\frac{J}{S} \frac{\partial^{2} w_{b}}{\partial t^{2}}\right)=\frac{S}{D} \frac{\partial \vartheta}{\partial x} .
$$

Disturbing function on the right hand side in (37) can be ignored due to assumed uncoupling. Once $w_{b}$ is determined, the total beam deflection, according to (33), reads

$$
w=w_{b}-\frac{D}{S} \frac{\partial^{2} w_{b}}{\partial x^{2}}+\frac{J}{S} \frac{\partial^{2} w_{b}}{\partial t^{2}} .
$$

The right hand side of (34) represents differential equation of axial shear vibrations 


$$
\frac{\partial^{2} \vartheta}{\partial x^{2}}-\frac{S}{D} \vartheta-\frac{J}{D} \frac{\partial^{2} \vartheta}{\partial t^{2}}=0
$$

\subsection{General solution of flexural natural vibrations}

Natural vibrations are harmonic, i.e., $w_{b}=W_{b} \sin \omega t$ and $\vartheta=\Theta \sin \omega t$, so that equations of motion (37) and (39) are related to the vibration amplitudes

$$
\begin{gathered}
\frac{\mathrm{d}^{4} W_{b}}{\mathrm{~d} x^{4}}+\omega^{2}\left(\frac{J}{D}+\frac{m}{S}\right) \frac{\mathrm{d}^{2} W_{b}}{\mathrm{~d} x^{2}}+\omega^{2} \frac{m}{D}\left(\omega^{2} \frac{J}{S}-1\right) W_{b}=0 \\
\frac{\mathrm{d}^{2} \Theta}{\mathrm{d} x^{2}}+\frac{S}{D}\left(\omega^{2} \frac{J}{S}-1\right) \Theta=0
\end{gathered}
$$

Amplitude of total deflection, according to (38), reads

$$
W=\left(1-\omega^{2} \frac{J}{S}\right) W_{b}-\frac{D}{S} \frac{\mathrm{d}^{2} W_{b}}{\mathrm{~d} x^{2}}
$$

Eq. (40) is known in literature as an reliable alternative of Timoshenko differential equations, (Senjanović and Fan 1989, Senjanović et al. 2009, Li 2008).

By comparing (40) with (9) it is obvious that differential equation of flexural vibrations of the modified beam theory is of the same structure as that of Timoshenko beam theory, but they are related to different variables, i.e., $W$ and $W_{b}$ deflection, respectively. Therefore, general solution for $W$ presented in Section 2.2 is valid for $W_{b}$ with all derivatives. In that case flexural displacements and sectional forces read

$$
\begin{gathered}
W=B_{1}\left(1-\omega^{2} \frac{J}{S}-\alpha^{2} \frac{D}{S}\right) \operatorname{sh} \alpha x+B_{2}\left(1-\omega^{2} \frac{J}{S}-\alpha^{2} \frac{D}{S}\right) \operatorname{ch} \alpha x \\
+B_{3}\left(1-\omega^{2} \frac{J}{S}+\beta^{2} \frac{D}{S}\right) \sin \beta x+B_{4}\left(1-\omega^{2} \frac{J}{S}+\beta^{2} \frac{D}{S}\right) \cos \beta x \\
\Phi=-\frac{\mathrm{d} W_{b}}{\mathrm{~d} x}=-\left(B_{1} \alpha \operatorname{ch} \alpha x+B_{2} \alpha \operatorname{sh} \alpha x+B_{3} \beta \cos \beta x-B_{4} \beta \sin \beta x\right) \\
M=-D \frac{\mathrm{d}^{2} W_{b}}{\mathrm{~d} x^{2}}=-D\left(B_{1} \alpha^{2} \operatorname{sh} \alpha x+B_{2} \alpha^{2} \operatorname{ch} \alpha x-B_{3} \beta^{2} \sin \beta x-B_{4} \beta^{2} \cos \beta x\right) \\
Q=-D \frac{\mathrm{d}^{3} W_{b}}{\mathrm{~d} x^{3}}-\omega^{2} J \frac{\mathrm{d} W_{b}}{\mathrm{~d} x}=-D\left[B_{1} \alpha\left(\alpha^{2}+\omega^{2} \frac{J}{D}\right) \operatorname{ch} \alpha x+B_{2} \alpha\left(\alpha^{2}+\omega^{2} \frac{J}{D}\right) \operatorname{sh} \alpha x-\right. \\
\left.-B_{3} \beta\left(\beta^{2}-\omega^{2} \frac{J}{D}\right) \cos \beta x+B_{4} \beta\left(\beta^{2}-\omega^{2} \frac{J}{D}\right) \sin \beta x\right]
\end{gathered}
$$




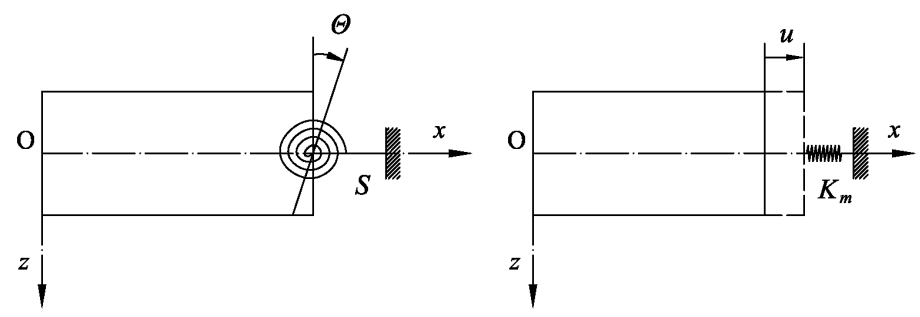

Fig. 2 Analogy between axial shear model and stretching model

Parameters $\alpha$ and $\beta$ are specified in Section 2.2, Eqs. (14) and (15), respectively.

In this case also parameter $\alpha$ can be zero that gives $\omega_{0}=\sqrt{S / J}$ and $\beta_{0}=\sqrt{(S / D)+(m / J)}$. By taking this fact into account, bending deflection $W_{b}$ is of the form (21), while total deflection according to (43), reads

$$
W=B_{1}^{*} x+B_{2}^{*}+\frac{D}{S} \beta_{0}^{2}\left(B_{3} \sin \beta_{0} x+B_{4} \cos \beta_{0} x\right)
$$

where $B_{1}^{*}$ and $B_{2}^{*}$ are new integration constants instead of $B_{1}$ and $B_{2}$, which are infinite due to zero coefficients.

Concerning the higher order frequency spectrum the governing expressions for displacements and forces, Eqs. (43)-(46), have to be transformed in the same manner as explained in Section 2.2.

\subsection{General solution of axial shear natural vibrations}

Differential Eq. (41) for natural axial shear vibrations of beam reads

$$
\frac{\mathrm{d}^{2} \Theta}{\mathrm{d} x^{2}}+\left(\omega^{2} \frac{J}{D}-\frac{S}{D}\right) \Theta=0
$$

It is similar to the equation for rod stretching vibrations

$$
\frac{\mathrm{d}^{2} u}{\mathrm{~d} x^{2}}+\omega_{R}^{2} \frac{m}{E A} u=0 .
$$

Difference is additional moment $S \Theta$, which is associated to inertia moment $\omega^{2} J \Theta$, and represents reaction of an imagined rotational elastic foundation with stiffness equal to the shear stiffness $S$, as shown in Fig. 2.

Solution of (49) and corresponding axial force $N=E A \frac{\mathrm{d} u}{\mathrm{~d} x}$ read

$$
\begin{gathered}
u=C_{1} \sin \chi x+C_{2} \cos \chi x \\
N=E A\left(C_{1} \chi \cos \chi x-C_{2} \chi \sin \chi x\right)
\end{gathered}
$$

where $\chi=\omega_{R} \sqrt{m /(E A)}$. Based on analogy between (48) and (49) one can write for shear slope angle and moment 


$$
\begin{gathered}
\Theta=C_{1} \sin \eta x+C_{2} \cos \eta x \\
M=D\left(C_{1} \eta \cos \eta x-C_{2} \eta \sin \eta x\right)
\end{gathered}
$$

where

$$
\eta=\sqrt{\omega^{2} \frac{J}{D}-\frac{S}{D}}
$$

Between natural frequencies of axial shear beam vibrations and stretching vibrations there is relation $\omega^{2}=\omega_{0}^{2}+\omega_{R}^{2}$, where $\omega_{0}=\sqrt{S / J}$ belongs to the axial shear mode obtained from (44), $\Theta=A_{0}+A_{1} x$, (which associates on sheared set of playing cards). It is interesting that $\omega_{0}$ is at the same time threshold frequency of flexural vibrations, as explained in Section 2.2.

\subsection{Simply supported beam}

Let us consider symmetric modes for which $A_{1}=A_{3}=0$, and boundary conditions $W(l / 2)=0$ and $M(l / 2)=0$. By employing formulae (43) and (45) one obtains frequency equation in the form

$$
\operatorname{Det}=\left(1-\omega^{2} \frac{J}{S}\right)\left(\alpha^{2}+\beta^{2}\right) \operatorname{ch} \alpha \frac{l}{2} \cos \beta \frac{l}{2}=0 .
$$

It includes additional factor comparing to (25) based on Timoshenko beam theory, from which threshold frequency $\omega_{0}=\sqrt{S / J}$ is determined. Since the remained part of (55) is identical to (25), everything what is written in Section 2.3 is valid in this case including formula (29) for natural frequencies. If double frequency phenomenon is analysed in the same way as that for Timoshenko beam, the same results are obtained.

\subsection{Clamped beam}

Boundary conditions read $W=0$ and $\Phi=0$ at $x= \pm l / 2$. By employing (43) and (44) one obtains frequency equations for the first response spectrum and symmetric and antisymmetric modes listed in Appendix B, Eqs. (B1) and (B2). In a similar way, after modification of Eqs. (43) and (44) for higher spectrum, the obtained frequency equations are presented by Eqs. (B3) and (B4).

\subsection{Free beam}

For a free beam $M=0$ and $Q=0$ at $x= \pm l / 2$. By employing (45) and (46) one obtains frequency equations for the first response spectrum and symmetric and antisymmetric modes shown in Appendix B, Eqs. (B5) and (B6). Frequency equations for higher spectrum, after modification of Eqs. (45) and (46), are represented by Eqs. (B7) and (B8).

\subsection{Axial shear vibrations}

A beam performing axial shear vibrations can be fixed or free at both ends, or one end can be fixed and another free. Mode function $\Theta_{n}=C \sin \eta_{n} x, \eta_{n}=n \pi / l, n=1,2 \ldots$ satisfies boundary conditions 
for fixed beam $\Theta(0)=\Theta(l)=0$. By taking into account Eq. (54) for $\eta$ one obtains expression for natural frequencies

$$
\omega_{n}=\sqrt{\frac{S}{J}+\frac{D}{J}\left(\frac{n \pi}{l}\right)^{2}} .
$$

If beam is free $M(0)=M(l)=0$, and frequency equation reads $\eta^{2} \sin \eta l=0$. The first condition $\eta=0$ gives according to (54) threshold frequency $\omega_{0}=\sqrt{S / J}$, while the second condition $\sin \eta l=0$ requires $\eta_{n} l=n \pi / l$. Hence, natural frequencies are represented by Eq. (56) and natural mode is $\Theta_{n}=C \sin \eta_{n} x$.

For combined fixed-free boundary conditions, $\Theta(0)=0$ and $M(l)=0$, frequency equation reads $\eta \cos \eta l=0$. Again, $\eta=0$ gives $\omega_{0}$ and $\cos \eta l=0$ requires $\eta_{n}=(2 n-1) \pi / 2 l, \mathrm{n}=1,2 \ldots$ Expression for natural frequencies reads

$$
\omega_{n}=\sqrt{\frac{S}{J}+\frac{D}{J}\left[\frac{(2 n-1) \pi}{2 l}\right]^{2}}
$$

Natural mode is $\Theta_{n}=C \sin \eta_{n} x$.

\section{Illustrative numerical examples}

\subsection{Simply supported beam}

A beam of I-profile with height-to-length ratio $h / l=0.2$ and shear coefficient $k=5 / 6$ is analysed. Due to reason of simplicity dimensionless frequency parameter $\lambda=\omega / \omega_{0}$ is introduced. Natural frequencies of flexural vibrations are given by (29) and frequency parameter can be presented in the form

$$
\lambda_{n}^{f(1,2)}=\frac{1}{\sqrt{2}} \sqrt{1+c_{n} \mp \sqrt{\left(1+c_{n}\right)^{2}-d_{n}}}
$$

where

$$
c_{n}=\left[1+\frac{2(1+v)}{k}\right] e_{n}, \quad d_{n}=\frac{8(1+v)}{k} e_{n}^{2}, \quad e_{n}=\frac{I}{A l^{2}}(n \pi)^{2} .
$$

Its values for the first and second frequency spectrum are listed in Table 1. They are the same for both Timoshenko beam theory (TBT) and modified theory (MBT).

In flexural vibrations of a simply supported beam, angle of rotation is free. Therefore, let us consider axial shear vibrations of free beam. Natural frequencies are given by (56) and frequency parameter can be presented in the form

$$
\lambda_{n}^{s}=\sqrt{1+\frac{2(1+v)}{k} e_{n}} .
$$


Table 1 Frequency parameter $\lambda=\omega / \omega_{0}$ of simply supported beam, $h / l=0.2$

\begin{tabular}{ccccc}
\hline \hline & \multicolumn{2}{c}{ Flexural, TBT and MBT } & \multicolumn{2}{c}{ Axial } \\
\cline { 2 - 5 } & $1^{\text {st }}$ spectrum, $\lambda_{n}^{f 1}$ & $2^{\text {nd }}$ spectrum, $\lambda_{n}^{f 2}$ & Stretching, $\lambda_{n}^{t}$ & Shear, $\lambda_{n}^{s}$ \\
\hline 0 & & $1.000^{*}$ & $1.000^{*}$ \\
1 & 0.055 & 1.064 & 0.320 & 1.050 \\
2 & 0.189 & 1.227 & 0.641 & 1.188 \\
3 & 0.362 & 1.445 & 0.961 & 1.387 \\
4 & 0.549 & 1.693 & 1.281 & 1.625 \\
5 & 0.741 & 1.959 & 1.602 & 1.888 \\
6 & 0.935 & 2.237 & 1.922 & 2.167 \\
$6.335^{*}$ & $1.000^{*}$ & & & \\
7 & 1.128 & 2.524 & 2.243 & 2.455 \\
8 & 1.321 & 2.816 & 2.563 & 2.751 \\
9 & 1.512 & 3.113 & 2.883 & 3.052 \\
10 & 1.702 & 3.414 & 3.204 & 3.356 \\
11 & 1.891 & 3.718 & 3.524 & 3.663 \\
12 & 2.079 & 4.024 & 3.844 & 3.972 \\
\hline
\end{tabular}

*Threshold

The second term in (60) belongs to the stretching vibrations and values for both parameters are listed in Table 1. Values of $\lambda_{n}^{t}$ are larger than $\lambda_{n}^{f 1}$ due to higher tensional than flexural stiffness. Both the second flexural spectrum, $\lambda_{n}^{f 2}$, and axial shear spectrum, $\lambda_{n}^{s}$, start with threshold parameter $\lambda_{0}=1$, and it is interesting that they are very close in spite of different number of modal nodes, Table 1.

\subsection{Clamped beam}

Values of natural frequencies for TBT in the lower and higher spectrum are determined by frequency equations (A1), (A2), (A3) and (A4) for symmetric and antisymmetric modes. Eqs. (B1), (B2), (B3) and (B4) are used for determining frequencies of MBT. Values of frequency parameters are equal for both TBT and MBT and are listed in Table 2. Frequency parameter for axial shear vibrations of fixed beam, which is equal to that of free beam is also listed in Table 2. In spite of the fact that $\lambda_{j}^{f H}$ and $\lambda_{n}^{s}$ start with the threshold value $\lambda_{0}=1$, they diverge for higher modes.

\subsection{Free beam}

Values of natural frequencies according to TBT and MBT are determined by Eqs. (A5), (A6), (A7) and (A8), and Eqs. (B5), (B6), (B7) and (B8), respectively. Values of frequency parameters are equal and are shown in Table 3, together with those for axial shear vibrations, which are the same as in the previous cases. 
Table 2 Frequency parameter $\lambda=\omega / \omega_{0}$ of clamped beam, $h / l=0.2, k=5 / 6$

\begin{tabular}{|c|c|c|c|}
\hline \multirow{2}{*}{$\begin{array}{l}\text { Mode no. } \\
\quad j\end{array}$} & \multicolumn{2}{|c|}{ Flexural TBT and MBT } & \multirow{2}{*}{ Axial shear, $\lambda_{j}^{s}$} \\
\hline & Lower spectrum, $\lambda_{j}^{f L}$ & Higher spectrum, $\lambda_{j}^{f H}$ & \\
\hline 0 & & & $1.000^{*}$ \\
\hline 1 & 0.106 & & 1.050 \\
\hline 2 & 0.242 & & 1.188 \\
\hline 3 & 0.404 & & 1.387 \\
\hline 4 & 0.577 & & 1.625 \\
\hline 5 & 0.758 & & 1.888 \\
\hline 6 & 0.941 & & 2.167 \\
\hline$*$ & $1.000 *$ & $1.000 *$ & \\
\hline 7 & & 1.066 & 2.455 \\
\hline 8 & & 1.123 & 2.751 \\
\hline 9 & & 1.235 & 3.052 \\
\hline 10 & & 1.314 & 3.356 \\
\hline 11 & & 1.451 & 3.663 \\
\hline 12 & & 1.508 & 3.972 \\
\hline
\end{tabular}

*Threshold

Table 3 Frequency parameter $\lambda=\omega / \omega_{0}$ of free beam, $h / l=0.2, k=5 / 6$

\begin{tabular}{cccc}
\hline \hline \multirow{2}{*}{$\begin{array}{c}\text { Mode no. } \\
j\end{array}$} & \multicolumn{2}{c}{ Flexural TBT and MBT } & Axial shear, $\lambda_{j}^{s}$ \\
\cline { 2 - 4 } 0 & Lower spectrum, $\lambda_{j}^{f L}$ & Higher spectrum, $\lambda_{j}^{f H}$ & $1.000^{*}$ \\
1 & 0.117 & & 1.050 \\
2 & 0.272 & & 1.188 \\
3 & 0.453 & & 1.387 \\
4 & 0.638 & & 1.625 \\
5 & 0.819 & & 1.888 \\
6 & 0.967 & & 2.167 \\
$*$ & $1.000^{*}$ & $1.000^{*}$ & \\
8 & & 1.070 & 2.455 \\
9 & & 1.097 & 2.751 \\
10 & & 1.272 & 3.052 \\
11 & & 1.279 & 3.356 \\
12 & & 1.299 & 3.663 \\
\hline
\end{tabular}

*Threshold

\section{Comparison of Timoshenko beam theory and modified beam theory}

\subsection{Natural frequencies}

Timoshenko beam theory deals with two differential equations of motion with two basic 
variables, i.e., deflection and angle of rotation. That system is reduced to one equation in terms of deflection and all physical quantities depend on its solution. On the other side, in the modified beam theory total deflection is split into pure bending deflection and shear deflection, while total angle of rotation consists of pure bending rotation and axial shear angle. The governing equations are condensed into single one for flexural vibrations with bending deflection as the main variable, and another for axial shear vibrations. Differential equations for flexural vibrations in both theories are of the same structure so that expressions for natural frequencies of simply supported beam are identical. Numerical examples show that values of natural frequencies for other boundary conditions are also the same, in spite of the fact that frequency equations are different. Such a result is not expected since for clamped Timoshenko beam boundary angle $\Psi=\Phi+\Theta=0$, while in the modified theory only $\Phi=0$. Hence, one could conclude that the Timoshenko beam theory will give somewhat higher frequency values than the modified theory due to fixation of the complete angle. Similar situation occurs in case of free beam, where total moment for Timoshenko beam $M_{\Psi}=M_{\Phi}+M_{\Theta}=0$, and in the modified theory $M_{\Phi}=0$.

Equal natural frequencies of flexural vibrations determined numerically can not be accepted as a rule. That fact should be confirmed in an analytical way. By comparing, for instance, frequency equations for clamped beam in lower spectrum and symmetric modes, Eqs. (A1) and (B1), they have the same functions but different coefficients. Since the equations give the same natural frequencies, their coefficients should be proportional. These equations can be written in matrix notation

$$
\left[\begin{array}{cc}
1-\omega^{2} \frac{J}{S}-\alpha^{2} \frac{D}{S} & 1-\omega^{2} \frac{J}{S}+\beta^{2} \frac{D}{S} \\
1-\frac{\omega^{2} m}{\beta^{2} S} & 1+\frac{\omega^{2} m}{\alpha^{2} S}
\end{array}\right]\left\{\begin{array}{c}
\beta \operatorname{ch} \alpha \frac{l}{2} \sin \beta \frac{l}{2} \\
\alpha \operatorname{sh} \alpha \frac{l}{2} \cos \beta \frac{l}{2}
\end{array}\right\}=\left\{\begin{array}{l}
0 \\
0
\end{array}\right\} .
$$

To meet the above condition of equal frequencies, determinant of the system (61) has to be zero. After some algebra determinant can be presented in the form

$$
\operatorname{Det}(\omega)=\frac{\alpha^{2}+\beta^{2}}{S^{2} \alpha^{2} \beta^{2}}\left(D S \alpha^{2} \beta^{2}-S m \omega^{2}+J m \omega^{4}\right) .
$$

By substituting Eqs. (14) and (15) for $\alpha$ and $\beta$ into (62) yields that the term in the brackets is zero.

In similar way one can prove that determinants of all pairs of frequency equations $\left(\mathrm{A}_{i}\right)$ and $\left(\mathrm{B}_{i}\right)$, $i=1,2 \ldots 8$ in Appendix A and B respectively, are zero. That is also valid for a beam with mixed boundary conditions, in which case complete expressions for displacements and forces with all four integration constants are taken into account.

\subsection{Natural modes}

Formulas for displacements and forces in TBT and MBT, Eqs. (17)-(20) and (39)-(42), respectively, are expressed with the same hyperbolic and trigonometric functions but their coefficients are different. Hence, it is necessary to compare mode shapes determined by TBT and MBT. For that purpose the previous example of clamped beam with symmetric modes in the lower frequency spectrum is taken into consideration.

Natural modes are characterized by shape, while their amplitude is arbitrary. From the first 
equation in (28) one finds

$$
A_{4}=-\frac{\operatorname{ch} \alpha \frac{l}{2}}{\cos \beta \frac{l}{2}} A_{2}
$$

where constant $A_{2}$ is chosen as referent one. In order to ensure the same total beam deflection within TBT and MBT, Eqs. (17) and (39), respectively, the following relations for integration constants in (39) have to be applied

$$
\begin{aligned}
& B_{2}=\frac{1}{1-\omega^{2} \frac{J}{S}-\alpha^{2} \frac{D}{S}} A_{2} \\
& B_{4}=\frac{1}{1-\omega^{2} \frac{J}{S}+\beta^{2} \frac{D}{S}} A_{4} .
\end{aligned}
$$

Numerical calculation of displacements and forces within TBT and MBT is performed for the following input data: $h=2 \mathrm{~m}, l=10 \mathrm{~m}, E=2.1 \cdot 10^{11} \mathrm{~N} / \mathrm{m}^{2}, v=0.3, \rho=7850 \mathrm{~kg} / \mathrm{m}^{3}$. Natural frequency is calculated from known frequency parameter $\lambda_{i}$ in Table 2, as $\omega_{j}=\omega_{0} \lambda_{j}$. Diagrams for total deflection, $W_{T}$ and $W_{M}$, angle of rotation, $\Psi$ and $\Phi$, bending moment, $M_{T}$ and $M_{M}$, and shear force, $Q_{T}$ and $Q_{M}$, for the first mode are shown in Fig. 3. Exactly the same values for TBT and MBT are obtained. Bending deflection $W_{b}$ and shear deflection $W_{s}$, determined within MBT, are also included in Fig. 3. Their boundary values are cancelled, resulting in zero edge total deflection. Shape of shear deflection mode is similar to that of bending moment, as result of their structure, Eqs. (38) and (41), respectively.

Diagrams of displacements and forces for the fifth mode determined by TBT and MBT, are shown in Fig. 4, and also are identical. Boundary values of bending deflection and shear deflection are quite large, but their sum is zero.

Equal displacement and force modes determined by TBT and MBT indicate that coefficients in corresponding equations are identical and this can be proved analytically. Let us compare, for instance, the second coefficient in the TBT shear force and that of MBT, Eqs. (20) and (42), respectively

$$
-\frac{\omega^{2} m}{\alpha} A_{2}=-D \alpha\left(\alpha^{2}+\omega^{2} \frac{J}{D}\right) B_{2} .
$$

By taking into account (64) the above relation can be presented in the form

$$
\alpha^{2}\left(\alpha^{2}+\omega^{2} \frac{J}{D}\right)-\omega^{2} m\left(1-\omega^{2} \frac{J}{S}-\alpha^{2} \frac{D}{S}\right)=0 .
$$

By substituting Eq. (14) for $\alpha$ into (67) all terms are cancelled.

Beam deflection $W$ is expressed with hyperbolic and trigonometric functions, Eq. (17). The latter are related to simply supported beam and the former compensate boundary influence, which is reduced to local effect for higher modes, as can be seen by comparing the first and fifth modes 

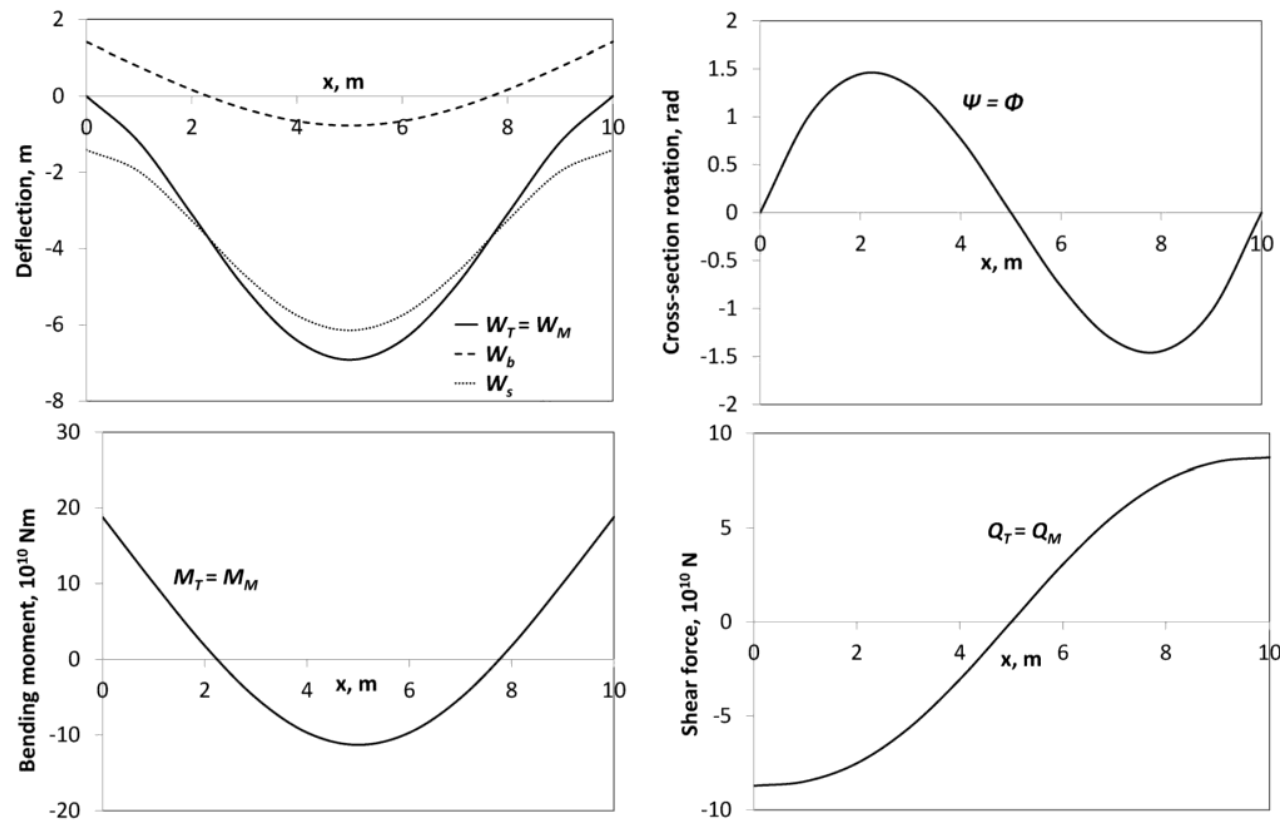

Fig. 3 The first flexural mode of clamped beam, $A_{2}=-1 \mathrm{~m}$
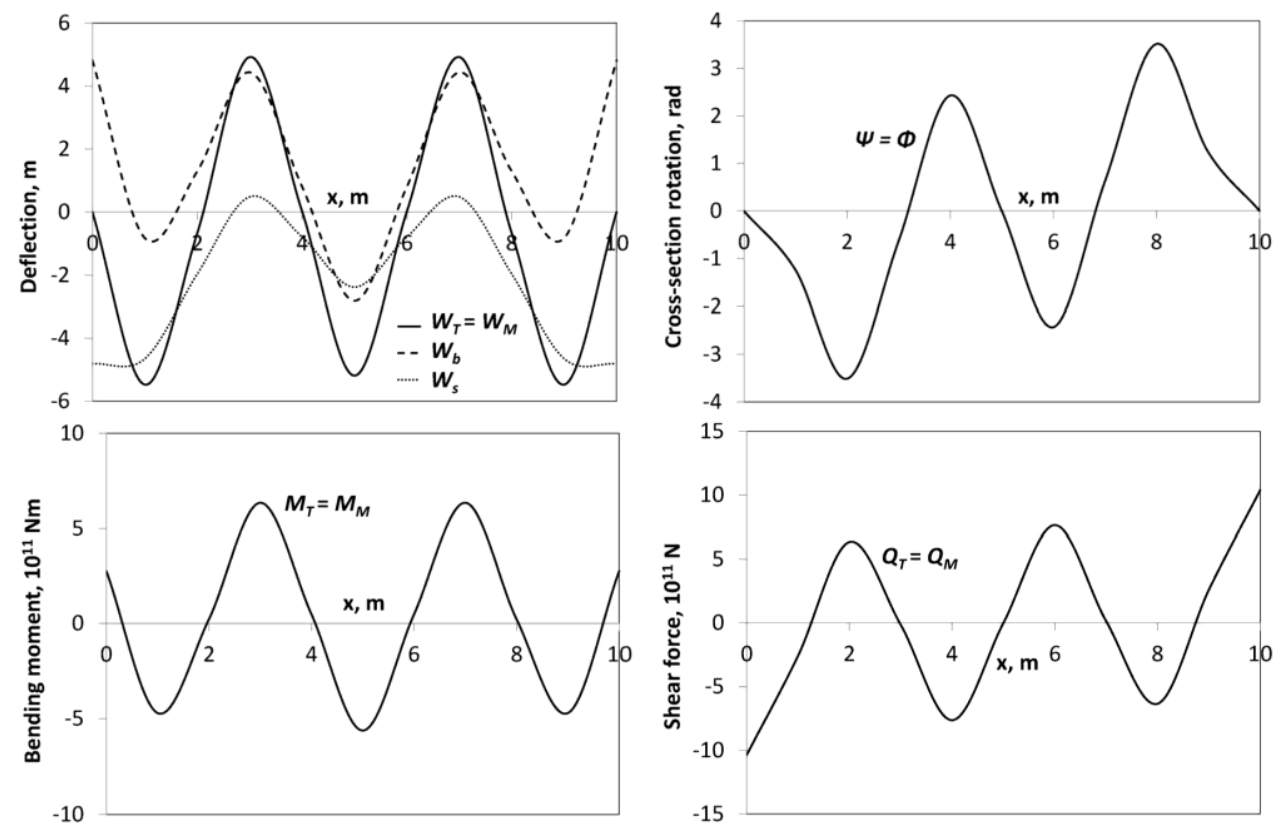

Fig. 4 The fifth flexural mode of clamped beam, $A_{2}=-0.1 \mathrm{~m}$

shown in Figs. 3 and 4. After threshold frequency $\omega_{0}$ boundary interference almost disappears and modes are expressed only with trigonometric functions as in the case of simply supported beam. Therefore, natural frequencies $\omega_{j}>\omega_{0}$ of clamped and free beam are very close, Tables 2 and 3 , 
and are of the same order of magnitude as those in the first frequency spectrum of simply supported beam, Table 1. Vibration parameters in arguments of trigonometric functions converge to the asymptotic value $\tilde{\alpha} \rightarrow \omega \sqrt{J / D}$ and $\beta \rightarrow \omega \sqrt{m / S}$ with frequency increased, as elaborated in Appendix C.

Axial shear vibrations are analysed within MBT assuming zero deflection. Their first mode occurs at threshold frequency $\omega_{0}$, which corresponds to transition flexural mode, Eq. (21), with a larger number of modal nodes where deflection is zero. Hence, assumption of uncoupled flexural and axial shear vibration is realistic. The same differential equation for axial vibration as (37) can be obtained in TBT from Eq. (4), by ignoring deflection.

\subsection{Static solution}

Comparison of TBT and MBT for static analysis is also interesting. One expects that expressions for static displacements can be obtained directly by deduction of dynamic expressions. In case of TBT static term of Eq. (9) leads to $W=A_{0}+A_{1} x+A_{2} x^{2}+A_{3} x^{3}$, and Eq. (10) gives $\Psi$ $=-\left(\mathrm{A}_{1}+2 A_{2} x+3 A_{3} x^{2}\right)$. That results in zero shear force $Q$, Eq. (1), and is also obvious from (20) if $\omega=0$ is taken into account. Therefore, in order to overcome this problem, it is necessary to return back to Eqs. (4) and (5) with static terms. By substituting (5) into (4), yields $D \mathrm{~d}^{3} \Psi / d x^{3}=0$, i.e., $\Psi$ $=-\left(A_{1}+2 A_{2} x+3 A_{3} x^{2}\right)$. Based on known $\Psi$, one obtains from (4)

$$
W=\frac{D}{S} \frac{\mathrm{d} \Psi}{\mathrm{d} x}-\int \Psi \mathrm{d} x+A_{0}=A_{0}+A_{1} x+A_{2} x^{2}+A_{3} x^{3}-\frac{2 D}{S}\left(A_{2}+3 A_{3} x\right) .
$$

On the other side, static part of Eq. (36) of MBT gives $W_{b}=B_{0}+B_{1} x+B_{2} x^{2}+B_{3} x^{3}$, and from (38) directly yields

$$
W=W_{b}-\frac{D}{S} \frac{\mathrm{d}^{2} W_{b}}{\mathrm{~d} x^{2}}=B_{0}+B_{1} x+B_{2} x^{2}+B_{3} x^{3}-\frac{2 D}{S}\left(B_{2}+3 B_{3} x\right)
$$

which is the same as (68). Angle of rotation is $\Phi=-\mathrm{d} W_{b} / d x=-\left(B_{1}+2 B_{2} x+3 B_{3} x^{2}\right)$ that is the same as the above $\Psi$ in TBT. If static solution for $W$ and $\Phi$, which are strongly dependent, is used for development of beam finite element shear locking, as mentioned in the Introduction, does not occur.

\section{Conclusions}

The research is motivated by the fact that an overall physical insight into Timoshenko beam theory has not been done after more than 90 years of its wide and successful application. The modified Timoshenko beam theory is result of such investigation. Based on the performed comparative analysis between the Timoshenko beam theory (TBT) and the modified beam theory (MBT), the following conclusions are drawn:

- TBT deals with two differential equations of motion with total deflection and rotation, which are condensed into single equation in terms of deflection.

- In MBT total deflection is split into pure bending deflection and transverse shear deflection, and total rotation is decomposed into bending angle and axial shear angle.

- MBT operates with two uncoupled differential equations of motion, one for flexural and 
another for axial shear vibrations in terms of pure bending deflection and axial shear angle, respectively.

- TBT and MBT flexural differential equations are of the same structure and give the same values of natural frequencies and mode shapes, not only for simply supported beam, but also for any combination of boundary conditions.

- Two flexural response spectra are obtained for simply supported beam by both theories, shifted for threshold frequency.

- For a beam with mixed boundary conditions lower frequency spectrum is obtained up to threshold frequency, and then higher spectrum is continued. Double frequency spectrum doesn't occur in this case.

- Natural modes of higher spectrum are sinusoidal as in case of simply supported beam, and influence of boundary conditions is considerably reduced.

- Threshold frequency depends on shear stiffness and mass moment of inertia, and its value is increased for more slender beams.

- Axial shear vibrations result with an additional frequency spectrum, which starts with threshold frequency. Differential equation for axial shear vibrations can also be extracted from Timoshenko equations by assuming zero deflection.

- MBT with its differential equation is already known in literature, as an approximate alternative of TBT developed under some assumption. The performed comparative analysis shows that introduced assumption actually represents the reality, and therefore MBT is rigorous theory as well as TBT.

- Moreover, MBT holds mathematical model of axial shear vibrations, extracted from TBT, which is not manifested in flexural response of Timoshenko beam since flexural and axial displacement fields are not coupled.

The obtained results within this investigation could have some impact on the other aspects of application of the Timoshenko beam theory, as referred in the Introduction, like beam on elastic foundation, beam stability, elastically connected multiple beams, thick plate, beam and plate finite elements, etc.

Timoshenko beam theory and its modification are the first order shear deformation theories. In future work it would be interesting to investigate possibility to extend the modified beam theory to the second order, as it is done for Timoshenko beam theory by Levinson (1981a, 1981b). High order shear deformation beam theory is important for instance for longitudinal strength analysis of multideck ships like Cruise Vessels. They are characterized with quite stiff hull up to main deck, and high and light superstructure, that manifests non-uniform profile of axial displacement of ship cross-section, (Senjanović and Tomašević 1999).

\section{Acknowledgments}

This paper is dedicated to the memory on Stephen Prokofievitch Timoshenko, distinguished scientist, who was Professor of Technical Mechanics at the University of Zagreb from 1920 to 1922, before he moved to the USA and finally joined to the Stanford University, and his famous beam theory published in 1921 and 1922, which is still challenging topic of investigation and subject of application in theory and practice. The investigation was supported by the National Research Foundation of Korea (NRF) grant funded by the Korea government (MSIP) through GCRC-SOP (No. 2011-0030013). 


\section{References}

Carrera, E., Giunto, G. and Petrolo M. (2011), Beam Structures, Classical and advanced Theories, John Wiley \& Sons Inc., New York, NY, USA.

Cowper, G.R. (1966), “The shear coefficient in Timoshenko's beam theory”, J. Appl. Mech., 33, 335-340.

De Rosa, M.A. (1995), "Free vibrations of Timoshenko beams on two-parametric elastic foundation", Comput. Struct., 57, 151-156.

Geist, B. and McLaughlin, J.R. (1997), "Double eigenvalues for the uniform Timoshenko beam”, Appl. Math. Letters, 10(3), 129-134.

Inman, D.J. (1994), Engineering Vibration, Prentice Hall, Inc., Englewood Cliffs, New Jersey.

Levinson, M. (1981a), “A new rectangular beam theory”, J. Sound Vib., 74(1), 81-87.

Levinson, M. (1981b), "Further results of a new beam theory", J. Sound Vib., 77(3), 440-444.

Li, X.F. (2008), "A unified approach for analysing static and dynamic behaviours of functionally graded Timoshenko and Euler-Bernoulli beams", J. Sound Vib., 32(5), 1210-1229.

Matsunaga, H. (1999), "Vibration and buckling of deep beam-columns on two-parameter elastic foundations", J. Sound Vib., 228, 359-376.

Mindlin, R.D. (1951), "Influence of rotary inertia and shear on flexural motions of isotropic elastic plates", J. Appl. Mech., 18(1), 31-28.

Pavazza, R. (2005), "Torsion of thin-walled beams of open cross-sections with influence of shear", Int. J. Mech. Sci., 47, 1099-1122.

Pavazza, R. (2007), Introduction to the Analysis of Thin-Walled Beams, Kigen, Zagreb, Croatia. (in Croatian)

Pilkey, W.D. (2002), Analysis and Design of Elastic Beams, John Wiley \& Sons Inc., New York, NY, USA.

Reddy, J.N. (1997), "On locking free shear deformable beam elements", Comput. Meth. Appl. Mech. Eng., 149, 113-132.

Senjanović, I. and Fan, Y. (1989), “A higher-order flexural beam theory”, Comput. Struct., 10, 973-986.

Senjanović, I. and Fan, Y. (1990), "The bending and shear coefficients of thin-walled girders", Thin-Wall. Struct., 10, 31-57.

Senjanović, I. and Fan, Y. (1993), "A finite element formulation of ship cross-sectional stiffness parameters", Brodogradnja, 41(1), 27-36.

Senjanović, I. and Tomašević, S. (1999), "Longitudinal strength analysis of a Cruise Vessel in early design stage", Brodogradnja, 47(4), 350-355.

Senjanović, I., Tomašević, S. and Vladimir, N. (2009), "An advanced theory of thin-walled structures with application to ship vibrations", Mar. Struct., 22(3), 387-437.

Simsek, M. (2011), "Forced vibration of an embedded single-walled carbon nanotube traversed by a moving load using nonlocal Timoshenko beam theory", Steel. Comp. Struct., 11(1), 59-76.

Sniady, P. (2008), "Dynamic response of a Timoshenko beam to a moving force", J. Appl. Mech., 75(2), 0245031-0245034.

Stojanović, V. and Kozić, P. (2012), "Forced transverse vibration of Rayleigh and Timoshenko double-beam system with effect of compressive axial load", Int. J. Mech. Sci., 60, 59-71.

Stojanović, V., Kozić, P. and Janevski, G. (2013), "Exact closed-form solutions for the natural frequencies and stability of elastically connected multiple beam system using Timoshenko and higher-order shear deformation theory", J. Sound Vib., 332, 563-576.

Timoshenko, S.P. (1921), "On the correction for shear of the differential equation for transverse vibration of prismatic bars", Phylosoph. Magazine, 41(6), 744-746.

Timoshenko, S.P. (1922), "On the transverse vibrations of bars of uniform cross section", Phylosoph. Magazine, 43, 125-131.

Timoshenko, S.P. (1937), Vibration Problems in Engineering, 2nd Edition, D. van Nostrand Company, Inc. New York, NY, USA.

van Rensburg, N.F.J. and van der Merve, A.J. (2006), "Natural frequencies and modes of a Timoshenko 
beam", Wave Motion, 44, 58-69.

Zhou, D. (2001), "Vibrations of Mindlin rectangular plates with elastically restrained edges using static Timoshenko beam functions with Rayleigh-Ritz method", Intl. J. Solids Struct., 38, 5565-5580. 


\section{Appendix A. frequency equations for Timoshenko beam theory}

Clamped beam:

Lower spectrum, symmetric modes

$$
\left(1-\frac{\omega^{2} m}{\beta^{2} S}\right) \beta \operatorname{ch} \alpha \frac{l}{2} \sin \beta \frac{l}{2}+\left(1+\frac{\omega^{2} m}{\alpha^{2} S}\right) \alpha \operatorname{sh} \alpha \frac{l}{2} \cos \beta \frac{l}{2}=0
$$

Lower spectrum, antisymmetric modes

$$
\left(1-\frac{\omega^{2} m}{\beta^{2} S}\right) \beta \operatorname{sh} \alpha \frac{l}{2} \cos \beta \frac{l}{2}-\left(1+\frac{\omega^{2} m}{\alpha^{2} S}\right) \alpha \operatorname{ch} \alpha \frac{l}{2} \sin \beta \frac{l}{2}=0
$$

Higher spectrum, symmetric modes

$$
\left(1-\frac{\omega^{2} m}{\beta^{2} S}\right) \beta \cos \tilde{\alpha} \frac{l}{2} \sin \beta \frac{l}{2}-\left(1-\frac{\omega^{2} m}{\tilde{\alpha}^{2} S}\right) \tilde{\alpha} \sin \tilde{\alpha} \frac{l}{2} \cos \beta \frac{l}{2}=0
$$

Higher spectrum, antisymmetric modes

$$
\left(1-\frac{\omega^{2} m}{\beta^{2} S}\right) \beta \sin \tilde{\alpha} \frac{l}{2} \cos \beta \frac{l}{2}-\left(1-\frac{\omega^{2} m}{\tilde{\alpha}^{2} S}\right) \tilde{\alpha} \cos \tilde{\alpha} \frac{l}{2} \sin \beta \frac{l}{2}=0
$$

Free beam:

Lower spectrum, symmetric modes

$$
\alpha^{3}\left(1+\frac{\omega^{2} m}{\alpha^{2} S}\right) \operatorname{ch} \alpha \frac{l}{2} \sin \beta \frac{l}{2}+\beta^{3}\left(1-\frac{\omega^{2} m}{\beta^{2} S}\right) \operatorname{sh} \alpha \frac{l}{2} \cos \beta \frac{l}{2}=0
$$

Lower spectrum, antisymmetric modes

$$
\alpha^{3}\left(1+\frac{\omega^{2} m}{\alpha^{2} S}\right) \operatorname{sh} \alpha \frac{l}{2} \cos \beta \frac{l}{2}-\beta^{3}\left(1-\frac{\omega^{2} m}{\beta^{2} S}\right) \operatorname{ch} \alpha \frac{l}{2} \sin \beta \frac{l}{2}=0
$$

Higher spectrum, symmetric modes

$$
\tilde{\alpha}^{3}\left(1-\frac{\omega^{2} m}{\tilde{\alpha}^{2} S}\right) \cos \tilde{\alpha} \frac{l}{2} \sin \beta \frac{l}{2}-\beta^{3}\left(1-\frac{\omega^{2} m}{\beta^{2} S}\right) \sin \tilde{\alpha} \frac{l}{2} \cos \beta \frac{l}{2}=0
$$

Higher spectrum, antisymmetric modes

$$
\tilde{\alpha}^{3}\left(1-\frac{\omega^{2} m}{\tilde{\alpha}^{2} S}\right) \sin \tilde{\alpha} \frac{l}{2} \cos \beta \frac{l}{2}-\beta^{3}\left(1-\frac{\omega^{2} m}{\beta^{2} S}\right) \cos \tilde{\alpha} \frac{l}{2} \sin \beta \frac{l}{2}=0
$$




\section{Appendix B. frequency equations for modified beam theory}

Clamped beam:

Lower spectrum, symmetric modes

$$
\left(1-\omega^{2} \frac{J}{S}-\alpha^{2} \frac{D}{S}\right) \beta \operatorname{ch} \alpha \frac{l}{2} \sin \beta \frac{l}{2}+\left(1-\omega^{2} \frac{J}{S}+\beta^{2} \frac{D}{S}\right) \alpha \operatorname{sh} \alpha \frac{l}{2} \cos \beta \frac{l}{2}=0
$$

Lower spectrum, antisymmetric modes

$$
\left(1-\omega^{2} \frac{J}{S}-\alpha^{2} \frac{D}{S}\right) \beta \operatorname{sh} \alpha \frac{l}{2} \cos \beta \frac{l}{2}-\left(1-\omega^{2} \frac{J}{S}+\beta^{2} \frac{D}{S}\right) \alpha \operatorname{ch} \alpha \frac{l}{2} \sin \beta \frac{l}{2}=0
$$

Higher spectrum, symmetric modes

$$
\left(1-\omega^{2} \frac{J}{S}+\tilde{\alpha}^{2} \frac{D}{S}\right) \beta \cos \tilde{\alpha} \frac{l}{2} \sin \beta \frac{l}{2}-\left(1-\omega^{2} \frac{J}{S}+\beta^{2} \frac{D}{S}\right) \tilde{\alpha} \sin \tilde{\alpha} \frac{l}{2} \cos \beta \frac{l}{2}=0
$$

Higher spectrum, antisymmetric modes

$$
\left(1-\omega^{2} \frac{J}{S}+\tilde{\alpha}^{2} \frac{D}{S}\right) \beta \sin \tilde{\alpha} \frac{l}{2} \cos \beta \frac{l}{2}-\left(1-\omega^{2} \frac{J}{S}+\beta^{2} \frac{D}{S}\right) \tilde{\alpha} \cos \tilde{\alpha} \frac{l}{2} \sin \beta \frac{l}{2}=0
$$

Free beam:

Lower spectrum, symmetric modes

$$
\left(1-\frac{\omega^{2} J}{\beta^{2} D}\right) \beta \operatorname{ch} \alpha \frac{l}{2} \sin \beta \frac{l}{2}+\left(1+\frac{\omega^{2} J}{\alpha^{2} D}\right) \alpha \operatorname{sh} \alpha \frac{l}{2} \cos \beta \frac{l}{2}=0
$$

Lower spectrum, antisymmetric modes

$$
\left(1-\frac{\omega^{2} J}{\beta^{2} D}\right) \beta \operatorname{sh} \alpha \frac{l}{2} \cos \beta \frac{l}{2}-\left(1+\frac{\omega^{2} J}{\alpha^{2} D}\right) \alpha \operatorname{ch} \alpha \frac{l}{2} \sin \beta \frac{l}{2}=0
$$

Higher spectrum, symmetric modes

$$
\left(1-\frac{\omega^{2} J}{\beta^{2} D}\right) \beta \cos \tilde{\alpha} \frac{l}{2} \sin \beta \frac{l}{2}-\left(1-\frac{\omega^{2} J}{\tilde{\alpha}^{2} D}\right) \tilde{\alpha} \sin \tilde{\alpha} \frac{l}{2} \cos \beta \frac{l}{2}=0
$$

Higher spectrum, antisymmetric modes

$$
\left(1-\frac{\omega^{2} J}{\beta^{2} D}\right) \beta \sin \tilde{\alpha} \frac{l}{2} \cos \beta \frac{l}{2}-\left(1-\frac{\omega^{2} J}{\tilde{\alpha}^{2} D}\right) \tilde{\alpha} \cos \tilde{\alpha} \frac{l}{2} \sin \beta \frac{l}{2}=0
$$




\section{Appendix C. analysis of vibration parameters}

Vibration parameters $\alpha, \tilde{\alpha}, \beta$ and $\eta$ in arguments of hyperbolic and trigonometric functions of beam response can be normalized in dimensionless form and presented as function of threshold frequency $\omega_{0}$ in order to analyse their relationship.

Beam parameters are the following:

$$
m=\rho A, \quad J=\rho I, \quad D=E I, \quad S=k G A=\frac{E A}{\mu}, \quad \mu=\frac{2(1+v)}{k} .
$$

By employing (C1) threshold frequency reads

$$
\omega_{0}=\sqrt{\frac{S}{J}}=\sqrt{\frac{E A}{\mu \rho I}} .
$$

Any frequency can be expressed as fraction of threshold frequency, i.e. $\omega=\lambda \omega_{0}$. Terms in Eq. (14) for $\alpha$ take the following form

$$
\begin{gathered}
\frac{m}{S} \pm \frac{J}{D}=(\mu \pm 1) \frac{\rho}{E} \\
\frac{4 m}{D \omega^{2}}=\frac{4 \mu}{\lambda^{2}}\left(\frac{\rho}{E}\right)^{2} .
\end{gathered}
$$

By substituting the above formulas into Eqs. (14) and (15) yields

$$
\left\{\begin{array}{c}
\alpha r \\
\beta r
\end{array}\right\}=\left\{\frac{\lambda}{\sqrt{2 \mu}} \sqrt{\sqrt{(\mu-1)^{2}+\frac{4 \mu}{\lambda^{2}}} \mp(\mu+1)}\right\}
$$

where $r=\sqrt{I / A}$ is the radius of gyration. In the case of threshold frequency $\omega=\omega_{0}, \lambda=\lambda_{0}=1$ and $\alpha_{0} r=0$, while

$$
\beta_{0} r=\sqrt{1+\frac{1}{\mu}}
$$

For very high frequencies $\lambda>>1$, both $\tilde{\alpha} r$ and $\beta r$ converge to the asymptotic values

$$
\tilde{\alpha}_{a} r=\frac{\lambda}{\sqrt{\mu}}, \quad \beta_{a} r=\lambda .
$$

In similar way parameter of axial shear vibrations can be presented in the form

$$
\eta=\sqrt{\omega^{2} \frac{J}{D}-\frac{S}{D}}=\sqrt{\left(\lambda^{2}-1\right) \frac{S}{D}} .
$$

By taking into account $(\mathrm{C} 1)$ one obtains 


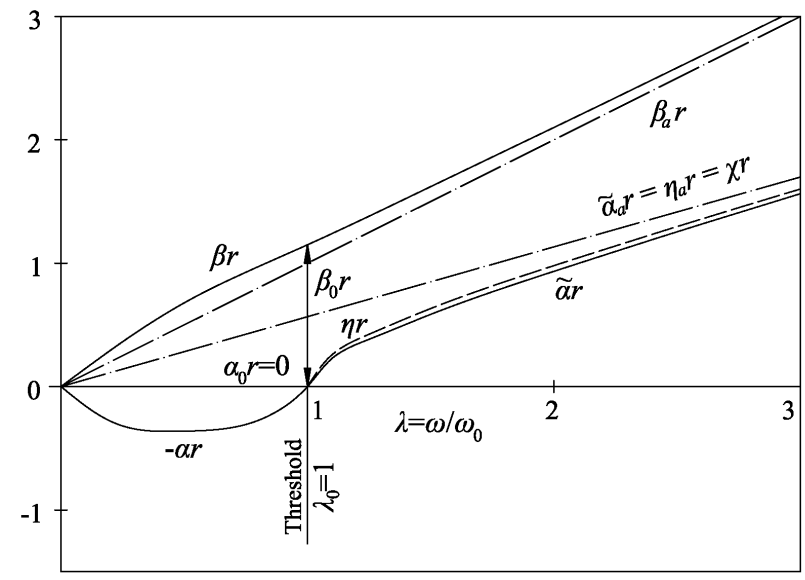

Fig. C1 Diagrams of beam vibration parameters

$$
\eta r=\sqrt{\frac{\lambda^{2}-1}{\mu}} .
$$

Asymptotic value of $\eta r$ is identical to that of $\tilde{\alpha} r$, Eq. (C7).

Vibration parameters for rod stretching vibration reads $\chi=\omega_{R} \sqrt{m /(E A)}$. By taking into account $\omega_{R}=\lambda \omega_{0}$ and $\omega_{0}=\sqrt{S / J}$, one obtains that $\chi r$ is identical to asymptotic value $\tilde{\alpha}_{a} r$, Eq. (C7).

Diagrams of dimensionless beam vibration parameters $\alpha r, \tilde{\alpha} r, \beta r, \eta r$ and $\chi r$ as function of $\lambda$ are shown in instructive Fig. C1. Parameter $\alpha r$ is transformed into $\tilde{\alpha} r$ at the threshold frequency, where $\alpha_{0} r=0$, while $\beta_{0} r$ is presented with (C6). Both $\tilde{\alpha} r$ and $\beta r$ converge to asymptotic values which are different. Parameter of axial shear vibrations $\eta r$ follows $\tilde{\alpha} r$, giving a close higher frequency spectrum.

A similar parametric analysis is performed by van Rensburg and van der Merve (2006), where flexural parameters $\alpha^{2}, \tilde{\alpha}^{2}$, and $\beta^{2}$ as functions of $\omega^{2}$ are shown. However, only slopes of their asymptotes are determined and indicated in corresponding figure of (van Rensburg and van der Merve 2006) in intuitive positions, which doesn't provide realistic insight into parameter convergence.

In general case natural frequencies are determined from frequency equation Det $=F(\alpha(\omega), \beta(\omega))=0$, which is formulated by satisfying boundary conditions. Step-by-step numerical procedure is used until such values of coupled vibration parameters $\alpha(\omega)$ and $\beta(\omega)$ meet the above condition. These values are distinct points in corresponding diagrams shown in Fig. C2 for clamped beam.

If beam is simply supported values of parameter pairs are known a priori

$$
(\beta r)_{n}=(\tilde{\alpha} r)_{n}=n \pi \frac{r}{l}
$$




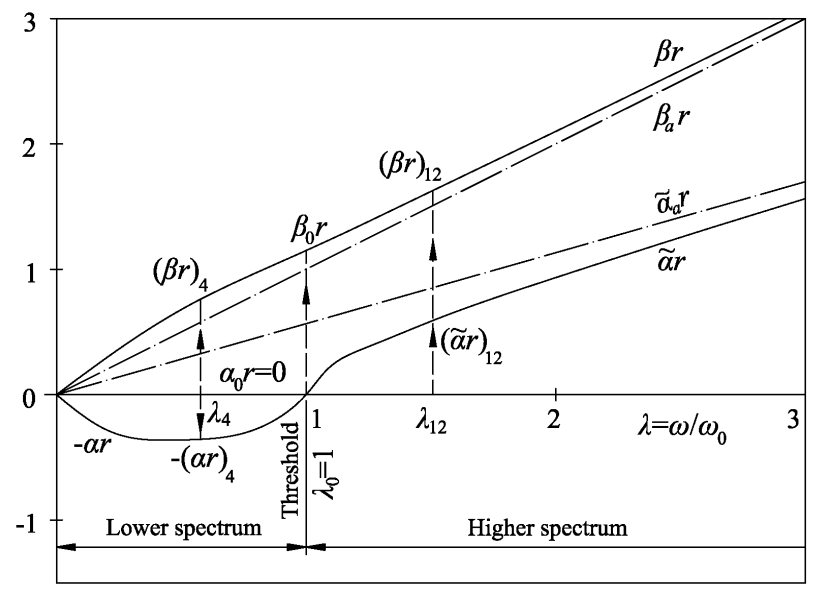

Fig. C2 Relations between vibration parameters and natural frequencies for clamped beam

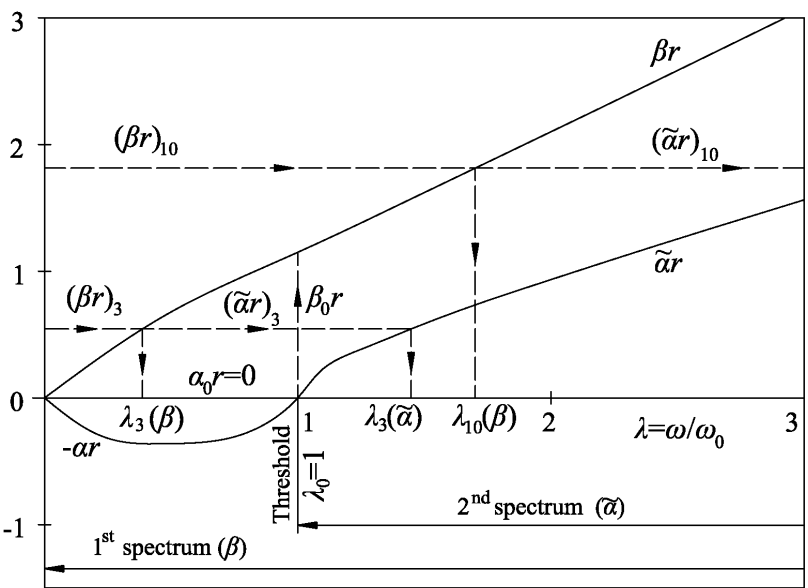

Fig. C3 Relations between vibration parameters and natural frequencies for simply supported beam

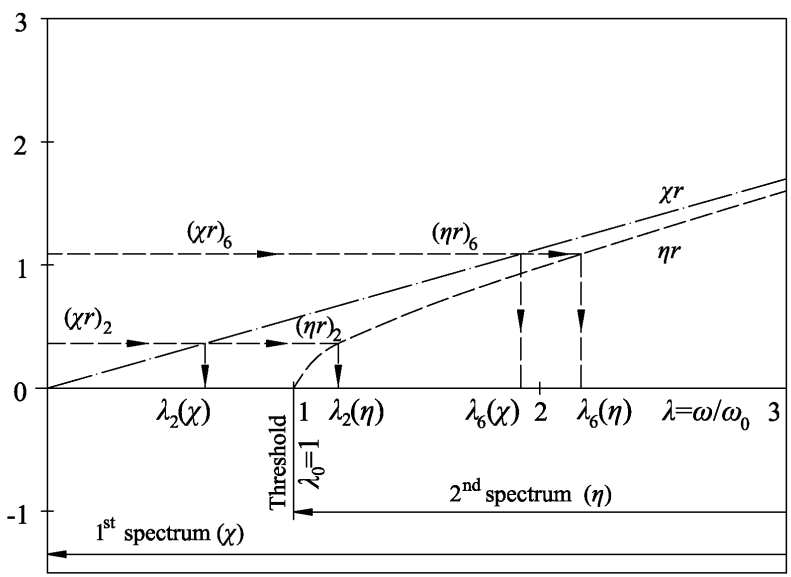

Fig. C4 Relations between vibration parameters and natural frequencies for axial vibrations 
By entering in parameter diagrams, natural frequencies for the first and second spectrum can be determined as shown in Fig. C3. Hence, for one value of $n$ there are two different frequencies but one mode shape.

In the above way it is proved in a physically transparent way that double frequency phenomenon is a characteristic of simply supported beam only.

Axial vibrations have also two spectra for any boundary conditions, one for stretching motion and another for shear motion. For given $n$, pairs of frequencies are obtained, also shown in Fig. C4. Corresponding natural modes are of the same shape, but of different physical meaning. 